\title{
De novo arteriovenous malformations following implantation of the HeartMate II left ventricular assist device
}

The HeartMate II (HMII) is the most popular continuous-flow left ventricular assist device (CF-LVAD) employed worldwide. Implantation of CF-LVADs has been associated with high rates of gastrointestinal bleeding, particularly from arteriovenous malformations (AVMs) [1,2]. Part of the increased bleeding risk relates to a reduction in circulating von Willebrand factor multimers [3]. However, it has also long been hypothesized that loss of the physiologic pulse, as occurs with CF-LVAD placement, may lead to hemodynamic changes in the gastrointestinal mucosa that are ultimately "angiodysplasia-genic" [4].

A 65-year-old man with no previous history of AVMs or gastrointestinal hemorrhage underwent uncomplicated implantation of a HMII device. He presented to the hospital 5 weeks postimplantation with frank hematochezia. After stabilization, he underwent both upper and lower gastrointestinal endoscopic evaluations, which revealed several large cecal AVMs with stigmata of recent bleeding ( $\bullet$ Fig.1), as well as numerous gastric antral AVMs without signs of bleeding. These AVMs were treated successfully with argon plasma coagulation (APC) therapy. He also underwent video capsule endoscopy, which revealed a single 4-mm, nonbleeding AVM in the jejunum, which was not treated. Once he had been stabilized, the patient was discharged home.

He re-presented to the hospital 4 weeks later with recurrent gastrointestinal bleeding. Repeat upper gastrointestinal endoscopy revealed the presence of two large gastric AVMs with active bleeding ( $\bullet$ Fig.2), which were treated with APC. Of note, these two AVMs had not been present on the previous endoscopy 4 weeks earlier. Despite treatment, the patient continued to experience gastrointestinal bleeding. ${ }^{99 \mathrm{~m}}$ Technetium-labeled red blood cell scintigraphy was performed, which revealed active bleeding in the jejunum consistent with the previously visualized AVM ( $\bullet$ Fig. 3).

Given the recurrent and diffuse nature of his AVMs across the entire gastrointestinal tract, he was treated with systemic pharmacologic therapy using octreotide. His gastrointestinal bleeding finally ceased after this octreotide therapy. He remains free of recurrent hemorrhage several months later.

\section{Endoscopy_UCTN_Code_CCL_1AC_2AB}

\section{Competing interests: None}

\section{R. J. Huang ${ }^{1}$, R. J. Wong ${ }^{2}$, K. V. Draper ${ }^{1}$, T. A. Winter ${ }^{2}$}

${ }^{1}$ Department of Medicine, Stanford University Medical Center, Stanford, California, USA

2 Division of Gastroenterology and Hepatology, Stanford University Medical Center, Stanford, California, USA

\section{References}

1 Stern DR, Kazam J, Edwards P et al. Increased incidence of gastrointestinal bleeding following implantation of the HeartMate II LVAD. J Card Surg 2010; 25: $352-356$

2 Demirozu ZT, Radovancevic R, Hochman LF et al. Arteriovenous malformation and gastrointestinal bleeding in patients with the HeartMate II left ventricular assist device. J Heart Lung Transplant 2011; 30: 849-853

3 Uriel N, Pak SW, Jorde UP et al. Acquired von Willebrand syndrome after continuousflow mechanical device support contributes to a high prevalence of bleeding during long-term support and at the time of transplantation. J Am Coll Cardiol 2010; 56: 1207-1213

4 Heyde EC. Gastrointestinal bleeding in aortic stenosis (Letter). N Engl J Med 1958; 259: 196

Bibliography

Dol http://dx.doi.org/

10.1055/s-0032-1325899

Endoscopy 2012; 44: E441

(c) Georg Thieme Verlag KG

Stuttgart · New York

ISSN 0013-726X

\section{Corresponding author}

R. J. Huang, MD

Stanford University

Medical Center

300 Pasteur Drive

Lane 154

Stanford, CA 94305-1509

USA

Fax: +1-650-723-4000

rjhuang@stanford.edu

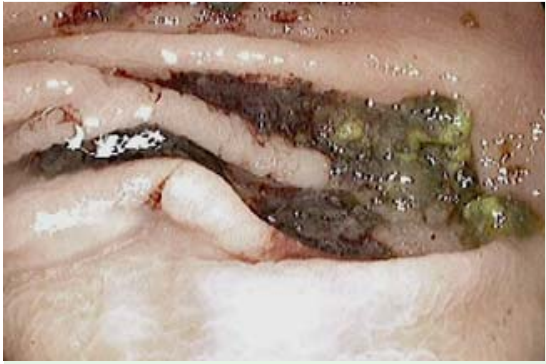

Fig. 1 Image from a lower gastrointestinal endoscopy performed on a 65-year-old man with a continuous-flow left ventricular assist device who presented with frank hematochezia showing several large cecal arteriovenous malformations with overlying clotted blood in the cecum.

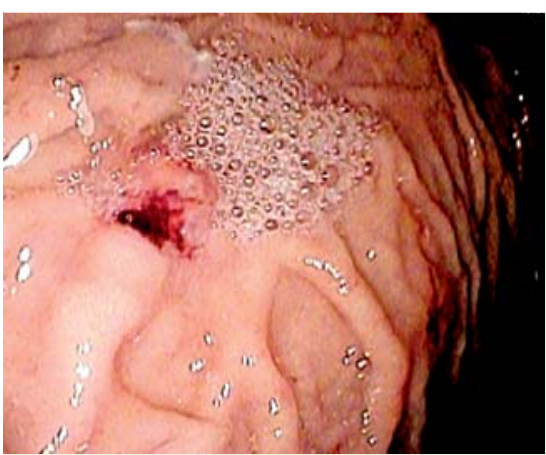

Fig. 2 Image from an endoscopy 4 weeks later when the patient re-presented with recurrent gastrointestinal bleeding showing the presence of two new 10-mm arteriovenous malformations in the proximal stomach that were not present on the previous endoscopic evaluation.

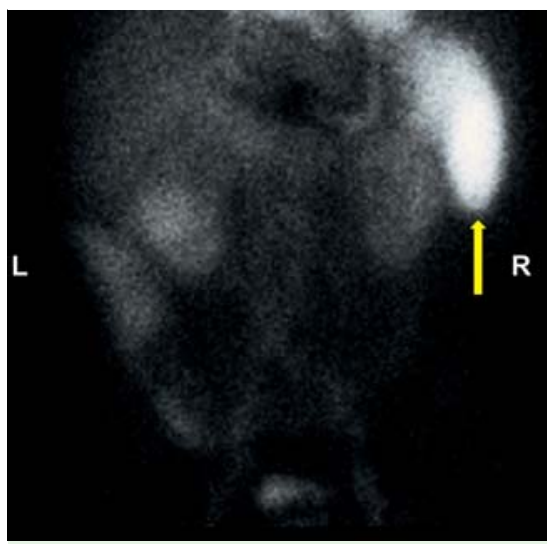

Fig. $399 \mathrm{~m}$ Technetium-labeled red blood cell scintigraphy demonstrating an abnormal focus of radiotracer in the left upper quadrant moving obliquely and inferiorly toward the midline (yellow arrow), suggestive of an actively bleeding jejunal lesion. 